\title{
A New Maximal Point Theorem
}

\author{
A. Göpfert and Chr. Tammer
}

\begin{abstract}
The aim of our paper is to generalize the maximal point theorem of Bishop and Phelps and to apply this result to derive a new multicriteria Ekeland's principle in a direct way by induction without making use of Ekeland's original scalar result.
\end{abstract}

Keywords: Maximal point theorem, conical supporting point, variational principle, approximation problem

AMS subject classification: $90 \mathrm{C} 26,49 \mathrm{~J} 40,90 \mathrm{C} 29$

\section{Introduction}

Suggested by the famous paper "On the variational principle" of Ekeland [8], many authors have published extensions and applications of Ekeland's variational principle as well as equivalent statements (cf. Borwein and Preiss [2], Brezis and Browder [3], Brondsted [4], Daneš [5], Figueiredo [6], Georgiev [9], Penot [20], Phelps [21], Rockafellar [22], Rolewicz [23] and Takahashi [24]). The results of Bishop and Phelps [1] from 1962 concerning supporting points of (convex) sets can also be seen as a first contribution to the topic of Ekeland's variational principle, given in the form of a maximal point theorem.

Even for the case in which the objective function takes its values in a partially ordered space, some authors have proved several variational principles. Loridan [16] derived a variational principle for vector optimization problems in finite-dimensional spaces. Nemeth [19] and Khanh [15] showed a general variational principle by using a cone-valued metric. Tammer [25] and Helbig and Pateva [7] proved variational principles via scalarization. Isac [13] presents an approach by using dynamical systems.

The aim of our paper is to prove a new maximal point theorem in a product space $X \times Z$, where $X$ and $Z$ are Banach spaces. This is a generalization of Phelps' maximal point theorem, which was shown in a product space $X \times \mathbb{R}$. In difference to Phelps' assertions it is possible to use our maximal point theorem in order to derive a variational principle for vector optimization problems in general spaces without any scalarization. This variational principle for vector optimization problems differs essentially from the variational principles given by Loridan [16], Nemeth [19], Khanh [15], Tammer [25],

A. Göpfert: Martin-Luther-Universität Halle - Wittenberg, FB Math./Inform., Universitätspl. 6, D - 06099 Halle

Chr. Tammer: Martin-Luther-Universität Halle - Wittenberg, FB Math./Inform., Universitätspl. 6, D - 06099 Halle 
Helbig and Pateva [7] and Isac [13] in the assumptions, in the assertions and in the proof.

We denote for a subset $B$ of a Banach space $Z$ the topological interior of the set $B$ by int $B$ and the topological closure of $B$ by $\mathrm{cl} B$. A functional $z: Z \rightarrow \mathbb{R}$ is called $B-$ monotone if $y^{1} \in y^{2}+B$ implies $z\left(y^{1}\right) \geq z\left(y^{2}\right)$ for all $y^{1}, y^{2} \in Z$. We say that a $B$-monotone functional $z$ is even strictly $B$-monotone if, additionally, $y^{1} \in y^{2}+(B \backslash\{0\})$ implies $z\left(y^{1}\right)>z\left(y^{2}\right)$.

Using the cone

$$
\mathcal{K}_{\varepsilon}^{\mathbb{R}}=\{(x, r) \in X \times \mathbb{R} \mid r+\sqrt{\varepsilon}\|x\| \leq 0\}
$$

where $\varepsilon$ is a parameter with $0<\varepsilon$ and $X$ is a Banach space, Phelps [21] showed that Ekeland's variational principle [8] is a direct consequence of a maximal point theorem. First let us recall Ekeland's and Phelps' results.

Theorem 1 (Ekeland [8]): Suppose that $\mathcal{B}$ is a closed subset of a Banach space $X$. Assume $f: \mathcal{B} \longrightarrow \overline{\mathbb{R}}$ is a proper, lower semicontinuous function, which is bounded from below, $\varepsilon>0$ and $\xi \in \mathcal{B}$ with $f(\xi) \leq \inf f+\varepsilon$. Then there exists an element $x^{0} \in \mathcal{B}$ such that

$$
\begin{aligned}
f\left(x^{0}\right) & \leq f(\xi)-\sqrt{\varepsilon}\left\|x^{0}-\xi\right\| \\
\left\|x^{0}-\xi\right\| & \leq \sqrt{\varepsilon} \\
f(x)+\sqrt{\varepsilon}\left\|x-x^{0}\right\| & >f\left(x^{0}\right) \quad \text { for all } x \neq x^{0} .
\end{aligned}
$$

Theorem 2 (Phelps [21]): Let $X$ be a Banach space and suppose $A \subset X \times \mathbb{R}$ is a non-empty closed set such that the set

$$
\{r \in \mathbb{R} \mid(x, r) \in A \text { for some } x \in X\}
$$

is bounded from below. Then for any point $(\xi, \beta) \in A$ there exists a point $\left(x^{0}, r^{0}\right) \in A$ such that

$$
\begin{gathered}
\left(x^{0} ; r^{0}\right) \in A \cap\left(\mathcal{K}_{\varepsilon}^{\mathbb{R}}+(\xi, \beta)\right) \\
\left\{\left(x^{0}, r^{0}\right)\right\}=A \cap\left(\mathcal{K}_{\varepsilon}^{\mathbb{R}}+\left(x^{0}, r^{0}\right)\right) .
\end{gathered}
$$

Theorem 2 simply means that under the given boundedness and closedness conditions for $A$ in the partial ordering (reflexive, transitive, antisymmetrical) defined by $\mathcal{K}_{\varepsilon}^{\mathbb{R}}$ any point $(\xi, \beta)$ of $A$ is dominated by at least one maximal point $\left(x^{0}, r^{0}\right)$ of $A$. It is also possible to interprete especially (4) or (6) in the form that $\left(x^{0}, f\left(x^{0}\right)\right)$ and $\left(x^{0}, r^{0}\right)$ solves the multicriteria optimization problems

$$
\begin{aligned}
& (x, r) \longrightarrow \max \quad \text { subject to }(x, r) \in \text { epigraph } f \\
& (x, r) \longrightarrow \max \text { subject to }(x, r) \in A
\end{aligned}
$$

respectively, where $X \times \mathbb{R}$ is partially ordered by $\mathcal{K}_{\varepsilon}^{\mathbb{R}}$. 
The first aim of our paper is to prove a new maximal point theorem, such that (5) and (6) hold even in $X \times Z$ ( $Z$ instead of $\mathbb{R}$ ), where $Z$ is a Banach space ordered by a given cone $K$. The point is to replace $\mathcal{K}_{e}^{\mathbb{R}}$ in (1) by an appropriate cone in $X \times Z$ (compare (9) in Section 2) and to evaluate a sequence of diameters of certain sets. Having proved such a maximal point theorem, a multicriteria Ekeland's principle follows. Furthermore, approximate efficiency can be interpreted in the same way as efficiency in (7), replacing $K$ in $Z$ by $\mathcal{K}_{\varepsilon}$ in $X \times Z$ (cf. (9) in Section 2). So, approximate solutions can be obtained by scanning epi $f$ in $X \times Z$ with help of $\mathcal{K}_{\varepsilon}$. Finally, some interesting relationships exist with supporting properties of sets.

Our paper is organized as follows. Section 2 deals with the new cone $\mathcal{K}_{\varepsilon}$, Section 3 gives the main theorem and its proof, Section 4 contains the multiobjective Ekeland's principle and some remarks. In Section 5 an example is presented.

\section{The cone $\mathcal{K}_{\varepsilon}$}

Let $X$ and $Z$ be Banach spaces, $Z$ partially ordered by a given cone $K \subset Z$, where $K$ is convex, pointed and closed with int $K \neq \emptyset$. Furthermore, choose $k^{0} \in$ int $K$ such that $\left\|k^{0}\right\|=1$. For any $\varepsilon$ with $0<\varepsilon$ we define the set

$$
\mathcal{K}_{\varepsilon}=\left\{(x, y) \in X \times Z \mid y+\sqrt{\varepsilon} k^{0}\|x\| \in-K^{r}\right\} .
$$

$\mathcal{K}_{\varepsilon}$ is clearly a cone, it is the hypograph of the mapping $-\sqrt{\varepsilon} k^{0}\|\cdot\|: X \longrightarrow Z$, recalling

$$
\text { hypo }\left(-\sqrt{\varepsilon} k^{0}\|\cdot\|\right):=\left\{(x, y) \mid y \in-\sqrt{\varepsilon} k^{0}\|\cdot\|-K\right\} \text {. }
$$

The cone $\mathcal{K}_{\epsilon}$ is pointed and closed, because $K$ has those properties. The triangle inequality implies that $\mathcal{K}_{\varepsilon}$ is convex and $\left(0,-k^{0}\right) \in \mathcal{K}_{\varepsilon}$. Finally, considering $K$ as embedded in $X \times Z$,

$$
K=\left\{(0,-y) \mid(x, y) \in \mathcal{K}_{e} \text { for some } x\right\}
$$

as we can easily see. Indeed,

$$
(x, y) \in \mathcal{K}_{e} \quad \text { implies } \quad y \in-\sqrt{\varepsilon} k^{0}\|x\|-K \subset-K
$$

and

$$
(0, y) \in\{0\} \times(-K) \text { implies } y+\sqrt{\varepsilon} k^{0}\|0\| \in-K
$$

that means $(0, y) \in \mathcal{K}_{e}$ for all $y \in-K$.

Taking $Z=\mathbb{R}, K=\mathbb{R}_{+}$and $k^{0}=1$ the set $\mathcal{K}_{e}$ defined by (9) coincides with the set $\mathcal{K}_{\varepsilon}^{\mathbb{R}}$ defined by (1). Given $\bar{y} \in-K$, the second inclusion in (9) is fulfilled for a norm-bounded set in $X$, otherwise there is a sequence $\left\{x_{n}\right\}$ with $\left\|x_{n}\right\| \longrightarrow \infty$, and so, for all $n$,

$$
-\left\|x_{n}\right\| k^{0} \in K+\frac{\bar{y}}{\sqrt{\varepsilon}} \quad \text { and } \quad-k^{0} \in K+\frac{1}{\left\|x_{n}\right\|} \frac{\bar{y}}{\sqrt{\varepsilon}}
$$


but $-k^{0} \notin K$.

Of course, conical support points are related to supporting points of convex sets (an overview can be found in Phelps [21]). Especially, if $X$ is a Banach space and $X^{*}$ its continuous dual, then taking $\gamma$ with $0<\gamma<1$ and $x^{*} \in X^{*}$ with $\left\|x^{*}\right\|=1$, the cone

$$
\mathcal{K}\left(x^{*} ; \gamma\right)=\left\{x \in X \mid \gamma\|x\| \leq x^{*}(x)\right\} \subset X
$$

is closed, linefree, convex and int $\mathcal{K}\left(x^{*}, \gamma\right) \neq \emptyset$. It is just the cone, which was used by Bishop and Phelps to prove density theorems for supporting points and functionals for convex sets (although the corresponding maximal point lemma for the supporting points does not use convexity). The cone (10) is related to that in (1) and (9) in an interesting way. For clarifying we consider the cone ( $\gamma$ and $X$ as above in (10))

$$
\mathcal{K}_{\gamma}^{X}=\left\{x \in X \mid x-\gamma k^{0}\|x\| \in K\right\}
$$

where $K \neq \emptyset$ is a convex, linefree cone in $X$ with $0 \in K$ and $\{0\} \neq K$, and $k^{0} \in K \backslash\{0\}$ with $\left\|k^{0}\right\|=1$. Then $\mathcal{K}_{\gamma}^{X}$ is clearly a cone, it is convex (because of the triangle inequality) and linefree. Obviously, $k^{0} \in \mathcal{K}_{\gamma}^{X}$ and if $k \in \mathcal{K}_{\gamma}^{X}$, then it follows

$$
k \in \gamma k^{0}\|k\|+K \subset K,
$$

that means $\mathcal{K}_{\gamma}^{X} \subset K$ and

a) if $\gamma \longrightarrow 0$, then $\mathcal{K}_{\gamma}^{X}$ approximates $K$

b) if $\gamma \longrightarrow 1$, then $\mathcal{K}_{\gamma}^{X}$ approximates the jet given by $k^{0}$.

Now we obtain the following two results relative to $\mathcal{K}_{\gamma}^{X}$ in (11):

(i) If $\bar{x} \in \mathcal{K}_{\gamma}^{X}$, then it follows $\bar{x}-\gamma k^{0}\|\bar{x}\| \in K$. Suppose $\bar{x}^{*} \in K^{*} \backslash\{0\}$ and $\bar{x}^{*}\left(k^{0}\right)=1$. Then it holds

$$
\bar{x}^{*}\left(\bar{x}-\gamma k^{*}\|\bar{x}\|\right) \geq 0 \quad \text { and therefore } \quad \bar{x}^{*}(\bar{x}) \geq \gamma\|\bar{x}\|
$$

that means, comparing with (10),

$$
\mathcal{K}_{\gamma}^{X} \subset \mathcal{K}\left(\bar{x}^{*}, \gamma\right)
$$

(ii) Using $\mathcal{K}_{\gamma}^{X}$ instead of $\mathcal{K}_{\varepsilon}$ in (9) leads to an other kind of maximal point theorems which generalize support. properties of sets (see [11]). In $\mathcal{K}_{\gamma}^{X}$ linear functionals $x^{*}$ do not appear, so contrary to results obtained with the help of $(10)$, corresponding conical supporting points (with help of (12)) do not only belong to the convex hull of $A$ but even to $A$ itself. 


\section{The main result}

Our main result is an assertion of the following kind: Under the assumption that a closed set $A \subset X \times Z$ satisfies certain boundedness condition, any point of $A$ is dominated by a maximal point, where we use the partial ordering defined by the cone $\mathcal{K}_{\varepsilon}$ (see (9)).

Theorem 3: Assume that $A$ is a closed subset of $X \times Z$, where $X$ and $Z$ are Banach spaces. Further, suppose that $K \subset Z$ is a pointed, closed, convex cone with non-empty interior, and assume

a) $k^{0} \in$ int $K$ and $\bar{y} \in Z$

b) $\{y \in Z \mid(x, y) \in A$ for some $x \in X\} \subset \bar{y}+K$

c) $B \subset Z$ is a set with $\operatorname{cl} B+(K \backslash\{0\}) \subset$ int $B$

d) $\varepsilon>0$.

Then for any point $(x, y) \in A$ there exists a point $\left(x_{0}, y_{0}\right) \in A$ such that

$$
\left(x_{0}, y_{0}\right) \in A \cap\left(\mathcal{K}_{e}+(x, y)\right) \quad \text { and } \quad\left\{\left(x_{0}, y_{0}\right)\right\}=A \cap\left(\mathcal{K}_{\varepsilon}+\left(x_{0}, y_{0}\right)\right) \text {. }
$$

Proof: Consider a sequence $\left\{A_{n}\right\}_{n \in \mathbb{N}}$ of sets

$$
A_{n}=A \cap\left(\mathcal{K}_{e}+\left(x_{n}, y_{n}\right)\right)
$$

Under the given assumptions the sets $A_{n}$ are closed. We define the sequence $\left\{\left(x_{n}, y_{n}\right)\right\}$ inductively as follows:

$$
\left(x_{1}, y_{1}\right)=(x, y)
$$

When we have obtained $\left(x_{1}, y_{1}\right),\left(x_{2}, y_{2}\right), \ldots,\left(x_{n}, y_{n}\right)$, then we choose $\left(x_{n+1}, y_{n+1}\right) \in A_{n}$ such that

$$
\nexists(x, y) \in A_{n} \quad \text { with } \quad y \in y_{n+1}-\frac{1}{n+1} k^{\circ}-(B \backslash\{0\}) .
$$

Such an element must exist because of the inclusion

$$
\{y \in Z \mid(x, y) \in A \text { for somé } x \in X\} \subset \bar{y}+K .
$$

The inclusion $\left(x_{n+1}, y_{n+1}\right) \in A_{n}$ implies the inclusion $\left(x_{n+1}, y_{n+1}\right) \in \mathcal{K}_{e}+\left(x_{n}, y_{n}\right)$ and so we can conclude that

$$
\mathcal{K}_{\varepsilon}+\left(x_{n+1}, y_{n+1}\right) \subset \mathcal{K}_{\varepsilon}+\mathcal{K}_{\varepsilon}+\left(x_{n}, y_{n}\right) \subset \mathcal{K}_{\varepsilon}+\left(x_{n}, y_{n}\right)
$$

where the last inclusion follows from the fact that $\mathcal{K}_{\varepsilon}$ is a cone. So we get from the definition of the sets $A_{n}$ in (13) that

$$
A_{n+1}=A \cap\left(\mathcal{K}_{\varepsilon}+\left(x_{n+1}, y_{n+1}\right)\right) \subset A \cap\left(\mathcal{K}_{e}+\left(x_{n}, y_{n}\right)\right)=A_{n}
$$

and hence

$$
A_{n+1} \subset A_{n} .
$$


Further, we have for an arbitrary element $(x, y) \in A_{n}$ the inclusion $(x, y) \in \mathcal{K}_{\varepsilon}+\left(x_{n}, y_{n}\right)$ such that the inclusions

$$
(x, y)-\left(x_{n}, y_{n}\right) \in \mathcal{K}_{\varepsilon} \quad \text { and } \quad y-y_{n}+\sqrt{\varepsilon}\left\|x-x_{n}\right\| k^{0} \in-K
$$

hold, and so we can conclude that

$$
y \in y_{n}-\sqrt{\varepsilon}\left\|x-x_{n}\right\| k^{0}-K .
$$

Because of (14) and the inclusion $(x, y) \in A_{n} \subset A_{n-1}$ we get the inequality $\sqrt{\varepsilon}\left\|x-x_{n}\right\|<$ $1 / n$ and so

$$
\left\|x-x_{n}\right\|<\frac{1}{\sqrt{\varepsilon} n} .
$$

On the other hand, we introduce a functional $z: Z \longrightarrow \mathbb{R}$ defined by

$$
z(y)=\inf \left\{t \in \mathbb{R} \mid y \in-\operatorname{cl} K+t k^{0}\right\}
$$

which is continuous, sublinear, strictly int $K$-monotone with the range $(-\infty, \infty)$ and $z\left(\alpha k^{0}\right)=\alpha$ for all $\alpha \in \mathbb{R}$ (compare [10] and [25]). Then for $(x, y) \in A_{n}$ from the sublinearity of the functional $z$ in (18) and from (14) and (15) it follows that

$$
z(y)+z\left(\frac{1}{n} k^{0}\right) \geq \inf _{\substack{y, w i t h \\(x, y) \in A_{n}}} z\left(y+\frac{1}{n} k^{0}\right) \geq \inf _{\substack{y, y \text { ith } \\(x, y) \in A_{n-1}}} z\left(y+\frac{1}{n} k^{0}\right) \geq z\left(y_{n}\right)
$$

and so $z(y)+z\left(\frac{1}{n} k^{0}\right) \geq z\left(y_{n}\right)$ such that

$$
z\left(y_{n}\right)-z(y) \leq \frac{1}{n}
$$

Moreover, it holds

$$
0 \leq z\left(y_{n}\right)-z(y)
$$

This we can show in the following way: For an arbitrary $(x, y) \in A_{n}$ because of (16) it holds

$$
y_{n}-y \in \sqrt{\varepsilon}\left\|x-x_{n}\right\| k^{0}+K \quad \text { and so } \quad y_{n}-\sqrt{\varepsilon}\left\|x-x_{n}\right\| k^{0} \in y+K \text {. }
$$

Further, the $K$-monotonicity of the functional $z$ in (18) (compare [10]) implies the inequality

$$
z\left(y_{n}-\sqrt{\varepsilon}\left\|x-x_{n}\right\| k^{0}\right) \geq z(y) .
$$

Taking into account the sublinearity of the functional $z$ we get

$$
z\left(y_{n}\right)+z\left(-\sqrt{\varepsilon}\left\|x-x_{n}\right\| k^{0}\right) \geq z(y) .
$$

So we can conclude

$$
z\left(y_{n}\right)-z(y) \geq \sqrt{\varepsilon}\left\|x-x_{n}\right\| \geq 0 \quad \text { and regarding (19) } \quad 0 \leq z\left(y_{n}\right)-z(y) \leq \frac{1}{n} .
$$


For $n \longrightarrow \infty$ we get $z\left(y_{n}\right)-z(y) \longrightarrow 0$. Because of (14) it follows from the definition of the functional $z$ in (18) that $y_{n}-y \longrightarrow 0$ as $n \longrightarrow \infty$ and hence $\left\|y_{n}-y\right\|<\delta_{n}$ for all $n, \delta_{n} \longrightarrow 0$ as $n \longrightarrow 0$. Using (17) we get

$$
\left\|x-x_{n}\right\|+\left\|y_{n}-y\right\| \leq \frac{1}{\sqrt{\varepsilon} n}+\delta_{n} \quad \text { and so } \quad \operatorname{diam} A_{n} \longrightarrow 0
$$

The completeness of $Z$ and the closedness of the sets $A_{n}$ yield that $\cap_{n} A_{n}$ contains only one element. Let be $\left(x_{0}, y_{0}\right)$ this element. Of course we have

$$
\left(x_{0}, y_{0}\right) \in A_{1}=A \cap\left(\mathcal{K}_{e}+(x, y)\right) \quad \text { which implies } \quad\left(x_{0}, y_{0}\right) \in \mathcal{K}_{\epsilon}+(x, y)
$$

Further,

$$
\left(x_{0}, y_{0}\right) \in A_{n}=A \cap\left(\mathcal{K}_{\varepsilon}+\left(x_{n}, y_{n}\right)\right) \quad(n \in \mathbb{N})
$$

implies

$$
\left(x_{0}, y_{0}\right) \in \mathcal{K}_{\varepsilon}+\left(x_{n}, y_{n}\right) \quad(n \in \mathbb{N})
$$

and so

$$
\left(x_{0}, y_{0}\right)+\mathcal{K}_{\varepsilon} \subset \mathcal{K}_{\varepsilon}+\left(x_{n}, y_{n}\right)+\mathcal{K}_{e} \subset \mathcal{K}_{\varepsilon}+\left(x_{n}, y_{n}\right) \quad(n \in \mathbb{N})
$$

Finally, we get

$$
A \cap\left(\mathcal{K}_{\varepsilon}+\left(x_{0}, y_{0}\right)\right) \subset A \cap\left(\mathcal{K}_{\varepsilon}+\left(x_{n}, y_{n}\right)\right)=A_{n} \quad(n \in \mathbb{N})
$$

and this implies $A \cap\left(\mathcal{K}_{e}+\left(x_{0}, y_{0}\right)\right)=\left\{\left(x_{0}, y_{0}\right)\right\}$

Remark 1: It is also possible to prove that diam $A_{n} \longrightarrow 0$ by using projections onto $k^{0}: P_{k^{\circ}} k=\alpha k^{0} \quad(\alpha \geq 0, k \in K)$.

\section{Conclusions}

Now we will show that a variational principle for an optimization problem with an objective function which takes its values in a partially ordered space is a direct consequence of our Theorem 3. The following Theorem 4 is an assertion about the existence of an efficient solution of a slightly perturbed vector optimization problem in a certain neighbourhood of an approximately efficient element of the original vector optimization problem.

In the following $X$ and $Z$ are considered to be real Banach spaces and $K \subset Z$ is a pointed, closed, convex cone with non-empty interior.

Now, we introduce a function $f: X \longrightarrow Z$ and assume that $f$ is bounded from below and lower semicontinuous with respect to $k^{0}$ and $K$. A function $f: X \longrightarrow Z$ is said to be bounded from below on $X$ if there exists an element $z \in Z$ with $f(X) \subset z+K$. We say that $f$ is lower semicontinuous with respect to $k^{0} \in$ int $K$ and $K$ (for short: $f$ is $\left(k^{0}, K\right)$-lower semicontinuous), if

$$
M_{r}^{X}=\left\{x \in X \mid f(x) \in r k^{0}-K\right\}
$$

is a closed set for each $r \in \mathbb{R}$. 
Remark 2: Put $Z=\mathbb{R}, K=\{z \in \mathbb{R} \mid z \geq 0\}, k^{0}=1$ and let be $M_{r}^{X}=\{x \in$ $X \mid f(x) \leq r\}$ a closed set for each $r \in \mathbb{R}$. In this special case our definition of $\left(k^{0}, K\right)$ lower semicontinuity coincides with the well known definition of lower semicontinuity for functionals $f: X \longrightarrow \mathbb{R}$.

Remark 3: Luc [18] uses the following cone-continuity concept: Let $K$ be a cone in $Z$. A function $f$ is $K$-semicontinuous if $\{x \in X \mid f(x) \in z-\operatorname{cl} K\}$ is a closed set at any point $z$ of $Z$. It is easy to see: If $K$ is a closed cone, then every $K$-semicontinuous function is $\left(k^{0}, K\right)$-lower semicontinuous.

Firstly, we will introduce approximately efficient elements of vector optimization problems. The reason for introducing approximately efficient solutions is the fact that numerical algorithms usually generate only approximative solutions anyhow and moreover that the efficient point set may be empty in the general non-compact case, whereas approximately efficient points always exist under very weak assumptions (see Tammer [26], where existence results for approximate solutions of a vector optimization problem were shown, especially under the assumption that the objective function is bounded from below).

Definition 1: An element $f\left(x_{\varepsilon}\right) \in f(X)$ is called an approximately efficient point of $f(X)$ with respect to $K, k^{0} \in \operatorname{int} K$ and $\varepsilon \geq 0$, if

$$
f(X) \cap\left(f\left(x_{\varepsilon}\right)-\varepsilon k^{0}-(K \backslash\{0\})\right)=\emptyset .
$$

The set of approximately efficient points of $f(X)$ wich respect to $K, k^{0}$ and $\varepsilon$ is denoted by

$$
\operatorname{Eff}\left(f(X), K_{e k^{0}}\right)
$$

where $K_{\varepsilon k^{0}}=\varepsilon k^{0}+K$. If we put $\varepsilon=0$, then the set $\operatorname{Eff}\left(f(X), K_{\varepsilon k^{0}}\right)$ coincides with the set of efficient points of $f(X)$ with respect to $K$, denoted by $\operatorname{Eff}(f(X), K)$.

Theorem 4: Assume that $f: X \longrightarrow Z$ is a $\left(k^{0}, K\right)$-lower semicontinuous function which is bounded from below. Then for any $\varepsilon>0$ and any $f\left(x^{0}\right) \in \operatorname{Eff}\left(f(X), K_{\varepsilon k^{0}}\right)$ there exists an element $x_{\varepsilon} \in X$ with the following properties:

1. $f\left(x_{\varepsilon}\right) \in f\left(x^{0}\right)-\sqrt{\varepsilon}\left\|x_{\varepsilon}-x^{0}\right\| k^{0}-K$

2. $\left\|x_{\varepsilon}-x^{0}\right\| \leq \sqrt{\varepsilon}$

3. $f_{\varepsilon k^{0}}\left(x_{\varepsilon}\right) \in \operatorname{Eff}\left(f_{\varepsilon k^{0}}(X), K\right)$, where $f_{\varepsilon k^{0}}(x):=f(x)+\sqrt{\varepsilon}\left\|x-x_{\varepsilon}\right\| k^{0}$.

Proof: The assumptions that $f$ is $\left(k^{0}, K\right)$-lower semicontinuous and int $K \neq \emptyset$ imply that the set

$$
\text { epi } f=\{(x, y) \in X \times Z \mid y \in f(x)+K\}
$$

is closed. Now, we put $A=$ epi $f$ and consider

$$
\mathcal{K}_{\varepsilon}+\left(x^{0}, f\left(x^{0}\right)\right)=\left\{(x, y) \in X \times Z \mid \sqrt{\varepsilon}\left\|x-x^{0}\right\| k^{0} \in-y+f\left(x^{0}\right)-K\right\} .
$$

Obviously, we have

$$
\left(x^{0}, f\left(x^{0}\right)\right) \in \text { epi } f \cap\left(\mathcal{K}_{e}+\left(x^{0}, f\left(x^{0}\right)\right) \neq \emptyset .\right.
$$


So the assumptions of Theorem 3 are fulfilled and we get a pair $\left(x_{\varepsilon}, y_{\varepsilon}\right)$ with

$$
\left(x_{\varepsilon}, y_{\varepsilon}\right) \in \text { epi } f \cap\left(\mathcal{K}_{\varepsilon}+\left(x^{0}, f\left(x^{0}\right)\right)\right.
$$

and

$$
\left\{\left(x_{e}, y_{e}\right)\right\}=\text { epi } f \cap\left(\mathcal{K}_{e}+\left(x_{e}, y_{e}\right)\right) .
$$

From (21) we get because of the inclusion $\left(x_{\varepsilon}, y_{\varepsilon}\right) \in$ epi $f$ that epi $f \cap\left(\mathcal{K}_{e}+\left(x_{\varepsilon}, f\left(x_{\varepsilon}\right)\right)=\right.$ $\left\{\left(x_{\varepsilon}, f\left(x_{\varepsilon}\right)\right)\right\}$. This means that there is no $x \in X, x \neq x_{\varepsilon}$ with

$$
(x, f(x)) \in \mathcal{K}_{e}+\left(x_{\varepsilon}, f\left(x_{\varepsilon}\right)\right)=\left\{(x, y) \mid y-f\left(x_{\varepsilon}\right)+\sqrt{\varepsilon}\left\|x-x_{\varepsilon}\right\| k^{0} \in-K\right\} .
$$

So we can conclude that

$$
f(x)-f\left(x_{\varepsilon}\right)+\sqrt{\varepsilon}\left\|x-x_{\varepsilon}\right\| k^{0} \notin-K \quad \text { for all } x \in X, x \neq x_{\varepsilon},
$$

which implies that for all $x \in X$ it holds

$$
f_{\varepsilon k^{\circ}}(X) \cap\left(f_{\varepsilon k^{0}}\left(x_{\varepsilon}\right)-(K \backslash\{0\})\right)=\emptyset,
$$

where

$$
f_{\varepsilon k^{\circ}}(x):=f(x)+\sqrt{\varepsilon}\left\|x-x_{\varepsilon}\right\| k^{0} .
$$

Hence, we get $f_{\varepsilon k^{\circ}}\left(x_{\varepsilon}\right) \in \operatorname{Eff}\left(f_{\varepsilon k^{\circ}}(X), K\right)$ and so we have shown that statement 3 holds.

Moreover, (20) implies

$$
\sqrt{\varepsilon}\left\|x_{\varepsilon}-x^{0}\right\| k^{0} \in f\left(x^{0}\right)-y_{\varepsilon}-K \quad \text { with } y_{\varepsilon} \in f\left(x_{\varepsilon}\right)+K
$$

and so we get

$$
\sqrt{\varepsilon}\left\|x_{\varepsilon}-x^{0}\right\| k^{0} \in f\left(x^{0}\right)-f\left(x_{\varepsilon}\right)-K-K
$$

and

$$
f\left(x_{\varepsilon}\right) \in f\left(\dot{x}^{0}\right)-\sqrt{\varepsilon}\left\|x_{\varepsilon}-x^{0}\right\| k^{0}-K
$$

which means that statement 1 is true.

Finally, for $f\left(x^{0}\right) \in \operatorname{Eff}\left(f(X), K_{\varepsilon k^{0}}\right)$ it holds

$$
\nexists x \in X: f(x) \in f\left(x^{0}\right)-\varepsilon k^{0}-(K \backslash\{0\}) .
$$

Taking into consideration statement 1 we get $\sqrt{\varepsilon}\left\|x_{\varepsilon}-x^{0}\right\| \leq \varepsilon$ and statement 2 .holds. This completes the proof

Remark 4: In [25] one of the authors proved a variational principle for optimization problems where the objective function takes its (more general) values in a linear toplogical space. In this proof we applied a separation theorem for non-convex sets and Ekeland's original result. In the proof of our Theorem 4 we don't use any scalarization of the vector optimization problem.

Remark 5: We can use the third assertion in Theorem 4 in order to prove necessary conditions for approximately efficient elements. Under differentiability assumptions we have shown that approximately efficient elements are solutions of approximate variational inequalities (compare Henkel and Tammer [12]). Moreover, for general approximation problems we have used the third statement in Theorem 4 in order to derive $\varepsilon$-Kolmogorov conditions for approximate solutions of vector-valued approximation problems (see [27]). 


\section{Example}

Finally, we will show, taking a vectorial approximation problem as example, that it is possible to use the third assertion in Theorem 4 in order to derive $\epsilon$-Kolmogorov conditions.

Let us assume that $\left(X,\|\cdot\|_{X}\right),\left(Z,\|\cdot\|_{Z}\right)$ and $\left(Y,\|\cdot\|_{Y}\right)$ are real reflexive Banach spaces.

Additionally to the assumptions of Theorem 4 in Section 4 we suppose that $K \subset Z$ is a closed convex cone with int $K \neq \emptyset$ having the. Daniell property, which means that every decreasing net $\left\{x_{i}\right\}_{i}$ (i.e. $i \leq j$ implies $x_{j} \leq x_{i}$ ) having a lower bound, converges to its infimum (see [14]). Further, we assume that $K$ has a weakly compact base.

In order to formulate our vector optimization problem, we will introduce a vectorvalued norm (compare [14]):

The mapping $\|\mid \cdot\|: Y \longrightarrow K$ is called a vector-valued norm if for all $y, y_{1}, y_{2} \in Y$ and for all $\lambda \in \mathbb{R}$ it holds:

1. $\||| y \mid\|=0 \Longleftrightarrow y=0$

2. ||$\lambda y\|||=|\lambda|\||y \||$

3. $\left\|\left|y_{1}+y_{2}\left\|\left|\in\left\|y_{1}\right\|\|+\|\left\|y_{2}\right\|\right|-K\right.\right.\right.$.

We suppose that $\||\cdot|\|$ is continuous.

The set of linear continuous mappings from $X$ to $Z$ is denoted by $L(X, Z)$. Suppose that $C \in L(X, Z), A \in L(X, Y)$ and $\alpha$ is a real non-negative number. Now, we consider the vector-valued function

$$
f(x):=C(x)+\alpha\|A(x)-a\| \|
$$

with $x \in X$ and $a \in Y$. Suppose that $f$ is bounded from below on $X$.

Now, we will derive necessary conditions for approximately efficient elements of the vectorial approximation problem

(P) Compute the set Eff $(f(X), K)$

by using the directional derivative of $f$ at $x \in X$ in the direction $v \in X$ :

$$
f^{\prime}(x)(v)=\lim _{t \rightarrow+0} \frac{f(x+t v)-f(x)}{t} .
$$

Using the directional derivative of the vector-valued norm and Theorem 2.27 of [14] we can conclude from statement 3 in Theorem 4 that for any $\varepsilon>0$ and any approximately efficient element

$$
f\left(x^{0}\right) \in \operatorname{Eff}\left(f(X), K_{\varepsilon k^{0}}\right)
$$

there exists an element $x_{\varepsilon} \in X$ with

$$
f\left(x_{\epsilon}\right) \in f\left(x^{0}\right)-\sqrt{\epsilon}\left\|x_{\epsilon}-x^{0}\right\|_{X} k^{0}-K
$$




$$
\left\|x_{\varepsilon}-x^{0}\right\|_{X} \leq \sqrt{\varepsilon}
$$

such that for any direction $v$ at $x_{e}$ with $\|v\|_{X}=1$ there is a linear continuous mapping $M_{e} \in \mathcal{L}_{x_{\varepsilon}}$, where

$$
\mathcal{L}_{x_{*}}=\left\{\begin{array}{l|l}
M \in L(X, Z) & \begin{array}{l}
M\left(A\left(x_{\epsilon}\right)-a\right)=\left\|A\left(x_{\varepsilon}\right)-a\right\| \\
\text { and }\|y\|-M(y) \in K \forall y \in Y
\end{array}
\end{array}\right\}
$$

with

$$
M_{\varepsilon} A(v) \in\{M A(v)\}+K \quad \text { for all } M \in \mathcal{L}_{x_{\varepsilon}}
$$

and

$$
C(v)+\alpha M_{\varepsilon} A(v) \notin-\sqrt{\varepsilon} k^{0}-\text { int } K .
$$

Remark 6: Relation (25) can be considered as an $\varepsilon$-variational inequality or $\varepsilon$ Kolmogorov condition.

Remark 7: Putting $\varepsilon=\bar{\varepsilon}=0$ we have elements $x_{\bar{\varepsilon}}$ and $M_{\bar{\varepsilon}} \in \mathcal{L}_{x_{\varepsilon}}$ according to (22) - (24) such that in condition (25) it holds

$$
C(v)+\alpha M_{\bar{\epsilon}} A(v) \notin-\text { int } K
$$

for all directions $v$ at $x_{\bar{\varepsilon}}$ with $\|v\|_{X}=1$. For the special case $C=0$ and $A=I$ this is a well-known necessary condition for weakly efficient elements of a certain class of vector-valued approximation problems (see [14]). But it has already been pointed out that the assumptions of the variational principle (Theorem 4) by themselves do not guarantee that such an element $x_{\bar{\varepsilon}}$ exists.

\section{References}

[1] Bishop, E. and R. R. Phelps: The support functionals of a convex set. Proc. Symp. Pure Math. (Amer. Math. Soc.) 7 (1962), 27 - 35.

[2] Borwein, J. M. and D. Preiss: A smooth variational principle with applications to subdifferentiability and to differentiability of convex functions. Trans. Amer. Math. Soc. 303 (1987), $517-527$.

[3] Brezis, H. and F. E. Browder: A general principle on ordered sets in nonlinear functional analysis. Adv. Math. 21 (1976), 355 - 364.

[4] Brondsted, A.: On a lemma of Bishop and Phelps. Pac. J. Math. 55 (1974), 335 - 341.

[5] Danes, J.: A geometric theorem useful in nonlinear functional analysis. Boll. Un. Mat. Ital. 6 (1972), 369 - 375.

[6] De Figueiredo, D. G.: Lectures on the Ekeland's Variational Principle with Applications and Detours (Tata Res. Inst. Fund. Res. Lec. Math. Phys.: Vol. 81). Berlin et al: Springer - Verlag 1989.

[7] Dentscheva, D. and S. Helbig: On variational principles, level sets, well-posedness, and $\varepsilon$-solutions in vector optimization. In preparation.

[8] Ekeland, I.: On the variational principle. J. Math. Anal. Appl. 47 (1974), 324 - 353. 
[9] Georgiev, P. G.: The strong Ekeland variational principle, the strong drop theorem and applications. J. Math. Anal. Appl. 131 (1988), 1 - 21.

[10] Gerth (Tammer), Chr. and P. Weidner: Nonconvex separation theorems and some applications in vector optimization. J. Optim. Theory Appl. 67 (1990), 297 - 320.

[11] Göpfert, A, and Chr. Tammer: $\varepsilon$-approximate solutions and conical support points. A new maximal point theorem. ZAMM 75 (1995), 595 - 596.

[12] Henkel, E.-C: and Chr. Tammer: $\varepsilon$-variational inequalities in partially ordered spaces. In preparation.

(13) Isac, G.: A variant of Ekeland's principle for Pareto E-efficiency. In preparation.

[14] Jahn, J.: Mathematical Vector Optimization in Partially Ordered Linear Spaces (Methoden und Verfahren der Mathematischen Physik: Vol. 31). Frankfurt/Main - Bern - New York: Verlag P. Lang (1986).

[15] Khanh, P. O.: On Caristi-Kirk's theorem and Ekeland's variational principle for Pareto extrema. Preprint. Warsaw: Polish Acad. Sci., Inst. Math.: Preprint 357 (1986), 1 - 7.

[16] Loridan, P.: $\varepsilon$-solutions in vector minimization problems. J. Optim. Theory Appl. 43 (1984), $265-276$.

[17] Loridan, P.: An application of Ekeland's variational principle to generalized Stackelberg problems. Ricerche di Math. 42 (1993), $159-178$.

[18] Luc, D. T.: Theory of vector optimization (Lect. Notes Econ. Math. Systems: Vol. 319). Berlin - Heidelberg - New York: Springer-Verlag 1989.

[19] Nemeth, A. B.: A nonconvex vector minimization problem. Nonlin. Anal.: Theory, Methods and Appl. 10 (1986), 669 - 678.

[20]. Penot, J. P.: The drop theorem, the petal theorem and Ekeland's variational principle. Nonlin. Anal.: Theory, Methods and Appl. 10 (1986), 813 - 822.

[21] Phelps, R. R.: Convex Functions, Monotone Operators and Differentiability (2nd Ed.). Lect. Notes Math. 1364 (1993), 1 - 118.

[22] Rockafellar, R. T.: Directionally Lipschitzian functions and subdifferential calculus. Proc. London Math. Soc. 39 (1979), 331 - 355.

[23] Rolewicz, S.: On drop property. Studia Math. 85 (1987), 27 - 35.

[24] Takahashi, W.: Existence theorems generalizing fixed point theorems for multivalued mappings. In: Fixed point theory and applications (Pitman Res. Notes Math.: Vol. 252; eds.: J. B: Baillon and M. Thera). Harlow: Longman 1991, pp. $397-406$.

[25] Tammer, Chr.: A Generalization of Ekeland's principle. Optimization 25 (1992), 129 . 141.

[26] Tammer, Chr.: Existence results and necessary conditions for $\varepsilon$-efficient elements. In: Multicriteria Decision (eds.: B. Brosowski et al). Frankfurt/Main: Verlag P. Lang 1993, pp. $97-109$.

[27] Tammer, Chr.: Necessary conditions for approximately efficient elements. Appr. and Optim. (to appear).

Received 22.09.1994; in revised form 16.02.1995 